\title{
Comparative Study of Mixing Liquids and Powders in a Continuous Medium using CFD
}

\author{
Alberto Mario Fuentes Enamorado ${ }^{1}$, Juan Bernardo Restrepo Betancourt ${ }^{2}$ and Luis Guillermo \\ Obregon $^{2}$ \\ ${ }^{1}$ Research Group in Process Simulation and Coal Transformation, Chemical Engineering Program, Universidad del Atlántico, \\ Barranquilla, Colombia \\ ${ }^{2}$ Research Group on Sustainable Chemical and Biochemical Processes, Chemical Engineering Program, Universidad del Atlántico, \\ Barranquilla, Colombia
}

Received 22 February 2018; Accepted 5 November 2018

\begin{abstract}
Prediction of flow and mixing of powders is a difficult challenge that has not been solved yet. Different numerical methods have been used to find the solution to the mathematical models proposed, but there are no experiments to compare and validate the results. The method with the best approximation is the discrete element method (DEM). However, this method requires a high computational power. In this article, it was made a comparison between the experimental results obtained when mixing two powders in an oscillatory low shear mixing device, and the 3D simulation of the same equipment using liquids with the help of a commercial software of computational fluids dynamics, CFD. Experiments at different wall velocities and wall displacements were run. Results reveal that the mixing in solids and liquids present lots of similarities when working at low velocity. This velocity lets solids to stay in frictional regime and liquids in the laminar regime. A zone of no mixing at the bottom of the mixing cavity is presented in liquids which is similar to the triangular pattern of no mixing found in solids. The height of the no mixing zone depends on the time of mixing, and the displacement and velocity of the walls. Under laminar regime conditions, the Navier Stokes equations can be applied to the powders because the velocity profiles obtained are similar.
\end{abstract}

Keywords: Shear stress, speed profile, CFD, simulation

\section{Introduction}

In the last two decades, the exponential growth of technology and the increase in computer performance for the resolution of complex simulations that are close to reality have allowed industries to create more profitable processes with high quality in the final product. An example is the powder mixing process, which is a very important step in the pharmaceutical and food industries, where uniform mixing and low variability must be guaranteed [1, 2]. Many companies use empirical or heuristic approaches to estimate critical variables of their processes based on the analysis of the product at the end of the production lines. However, these industries aim to optimize these bottlenecks using more reliable procedures [3]. There are two ways in which powder mixing can be optimized. One is the use of sensors inside the mixers, such as special cameras that use near infrared or NIR spectroscopy [3-5] to detect the final point of the mixture. The other is trying to predict the behavior of powder flow using simulation with discrete element methods (DEM) [6-8] or continuous mechanics [9-11] to determine the necessary conditions that lead to the best mixing in the shortest time. When performing simulations with continuous media, many authors use constitutive equations based on the way they link the stress tensor and the rate of deformation.

*E-mail address: luisobregon@mail.uniatlantico.edu.co ISSN: $1791-2377 @ 2018$ Eastern Macedonia and Thrace Institute of Technology. All rights reserved. doi:10.25103/iestr.115.16
However, there is a great difference between the mixing of liquids and powders. The transport properties and the transfer of momentum in liquids are isotropic, and in powders, they are not isotropic [12-14].

In this research, it was done a series of mixing simulations of two liquids with similar physical properties, such as density and viscosity. Then, these simulations were compared to an experimental work where two non-cohesive powders were mixed easily with the application of shear stress $[15,16]$. It was done with the purpose of comparing the same process in two different states of matter and see the advantages and disadvantages of each one. It was analyzed the differences and similarities of the velocity profiles and the mass fractions. The comparison lets to know whether the equation of continuity can be used in granular materials to predict mixing of powders or not.

\section{Methodology}

\subsection{Geometry and system properties}

The geometry of the system used in this computational work is shown in Fig. 1. It consists of a cuboid, where the length (L) is $22.86 \mathrm{~cm}$, the thickness (W) is $2.79 \mathrm{~cm}$ and a height $(\mathrm{H})$ is $5.08 \mathrm{~cm}$.

This mixer works by the movement of two rough movable parallel walls. The movable walls are located, one in the ZX plane at $Y=0 \mathrm{~cm}$ and the other at $\mathrm{Y}=2.79 \mathrm{~cm}$. These walls move using the following periodic function: 
$v=\left\{\begin{array}{c}a \frac{c m}{s} \rightarrow \text { if } 0<t \sec <T / 2 \\ -a \frac{c m}{s} \rightarrow \text { if } T / 2<t \sec <T\end{array}\right.$

The initial separation of the liquids in the mixing process is shown in Fig. 2.

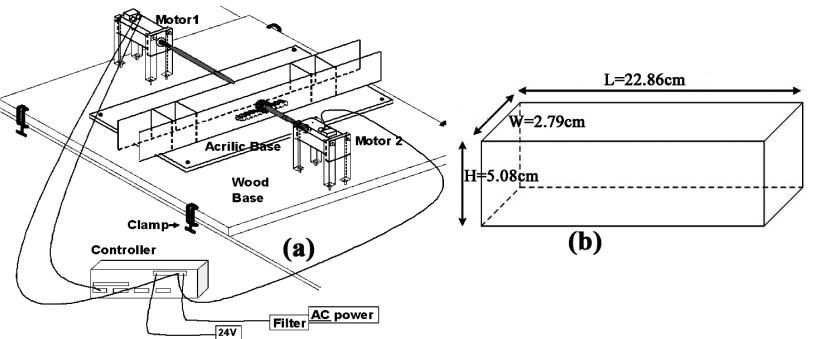

Fig. 1. (a) Diagram of the mixing system, (b) dimensions of the mixer cavity

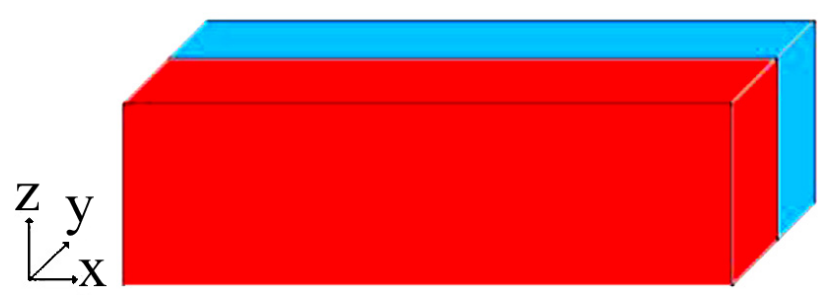

Fig. 2. Initial separation of the liquids before mixing

Regarding the properties of the two liquids, these are similar to the experiments with the mixing of powders, so it was used two Newtonian liquids with a density of 1400 $\mathrm{Kg} / \mathrm{m}^{3}$, viscosity of $1000 \mathrm{cP}$ and binary diffusivity of $10^{-8}$ $\mathrm{m}^{2} / \mathrm{s}$ [17]. It was used red and blue color to differentiate between the two liquids.

\subsection{Mathematical modeling}

To perform the simulation in an unsteady state and make an approximation to reality, it was used the momentum transport equation with the continuity equation corresponding to the system, see equations 2 to 7 [18].

\subsubsection{Continuity equation}

In this system, it was used the equation of continuity, Eq. 2.

$\frac{\partial \rho}{\partial t}+\nabla(\rho \overrightarrow{\boldsymbol{v}})=S_{m}$

Where $\nabla(\rho \overrightarrow{\boldsymbol{v}})$ is the density divergence of matter flow per unit volume, and $S_{m}$ is the mass added to the continuous phase from a second external dispersed phase, which is zero for this case. Eq. 2, is applicable only for one fluid, therefore, this equation must be modified so that it can be used for a number of components (NC), see Eq. 3.

$\frac{\partial}{\partial t}\left(\rho Y_{i}\right)+\nabla\left(\rho \overrightarrow{\boldsymbol{v}} Y_{i}\right)=-\nabla \overrightarrow{\boldsymbol{J}}_{i}+R_{i}+S_{i}$

where $Y_{i}$ is the mass fraction of component $i,-\nabla \vec{J}_{i}$ is the divergence of the diffusion flux of component $i, R_{i}$ is the rate of formation of component $i$, and $S_{i}$ is the addition of component $i$ from another external dispersed phase.

The last two terms of Eq. 3 are equal to zero, therefore:

$J_{i}=-\rho \mathcal{D}_{i, m} \nabla \boldsymbol{Y}_{i}-D_{T, i} \frac{\nabla \boldsymbol{T}}{\boldsymbol{T}}$
The term $\mathcal{D}_{i, m}$, corresponds to the diffusivity of component $i$ in the mixture:

$\mathcal{D}_{i, m}=\frac{1-Y_{i}}{\sum_{j=1, j \neq i}^{N C}\left(\frac{Y_{j}}{D_{i j}}\right)}$

The term on the right of Eq. 4 that corresponds to the thermal diffusion (Soret effect) is zero for our isothermal system. It is important to note that Eq. 4 refers to the balance of components and Eq. 2 to the global balance of matter for the control volume.

\subsubsection{Momentum balance}

In the simulations it was used the equation of motion, see Eq. 6:

$\frac{\partial}{\partial t}(\rho \overrightarrow{\boldsymbol{v}})+\nabla(\rho \overrightarrow{\boldsymbol{v}} \overrightarrow{\boldsymbol{v}})=\nabla \mathbf{P}+\nabla \boldsymbol{\tau}+\rho \overrightarrow{\boldsymbol{g}}+\overrightarrow{\boldsymbol{F}}$

Where $\nabla(\rho \overrightarrow{\boldsymbol{v}} \overrightarrow{\boldsymbol{v}})$ is the divergence of the momentum generated by the convective motion per unit volume, $\nabla \mathbf{P}$ is the pressure applied to the system, $\rho \overrightarrow{\boldsymbol{g}}$ is the force of gravity, $\overrightarrow{\boldsymbol{F}}$ is the external force applied to the control volume element and $\nabla \boldsymbol{\tau}$ is the divergence of the momentum generated by viscous effects or shear stresses per unit volume, see Eq. 7:

$\nabla \boldsymbol{\tau}=\mu\left[\left(\nabla \overrightarrow{\boldsymbol{v}}+\nabla \overrightarrow{\boldsymbol{v}}^{T}\right)-\frac{2}{3} \nabla \overrightarrow{\boldsymbol{v}} \mathbf{I}\right]$

Where "I" is the unitary tensor, and the last term on the right represents the dilatational viscosity of the fluid, which is negligible for Newtonian liquids.

\subsubsection{Simulation}

For the simulation, a commercial computational fluid mechanics software was used with a mesh-shaped with cubes of side $0.2325 \mathrm{~cm}$ generating 29601 nodes for the system, as shown in Fig. 3. The method for solving the differential equations was the finite volume method PISO (Pressure Implicit with Splitting of Operators) [19], where the iteration time step was $0.01 \mathrm{~s}$. This time was a result of the simulation because the software performs the necessary number of iterations until all the convergence criteria are met, e.g., the sum of the absolute residuals is less than the specified tolerances (usually values between $10^{-3}$ to $10^{-6}$ ).

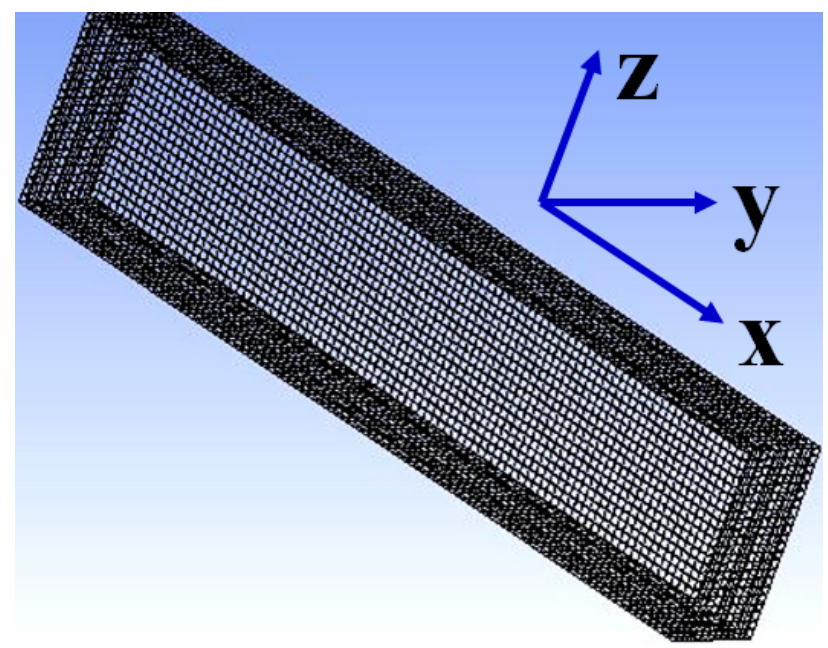

Fig. 3. Mesh of the mixer 
Alberto Mario Fuentes Enamorado, Juan Bernardo Restrepo Betancourt and Luis Guillermo Obregon/

Journal of Engineering Science and Technology Review 11 (5) (2018) 134 - 138

Fig. 1 was drawn in AutoCAD and imported to the CFD software. Each part of the mixer shown in Fig. 1 was imported separately so that the boundary conditions were given considering each part. The operating conditions of the system are (A) amplitude of displacement of the walls of $11.43 \mathrm{~cm}, 22.86 \mathrm{~cm}$ and $34.29 \mathrm{~cm}$, and wall velocities 13 $\mathrm{cm} / \mathrm{s}, 16 \mathrm{~cm} / \mathrm{s}$, and $66 \mathrm{~cm} / \mathrm{s}$. The duration of each oscillation (round trip) is shown in Tab. 1. The given friction at the top (contact air/liquid) was zero.

Table 1. Wall oscillation times

\begin{tabular}{c|c|c|c}
\hline \multirow{2}{*}{ Amplitude, cm } & \multicolumn{3}{|c}{ Wall velocity, cm/s } \\
\cline { 2 - 4 } & $\mathbf{1 3}$ & $\mathbf{1 6}$ & $\mathbf{6 6}$ \\
\hline $\mathbf{1 1 , 4 3}$ & 1,76 & 1,43 & 0,35 \\
\hline $\mathbf{2 2 , 8 6}$ & 3,52 & 2,86 & 0,69 \\
\hline $\mathbf{3 4 , 2 9}$ & 5,28 & 4,29 & 1,04 \\
\hline
\end{tabular}

The simulations were run at a temperature of $25^{\circ} \mathrm{C}$ and pressure of $101.32 \mathrm{kPa}$. For boundary conditions, two parallel walls move with the periodic function shown in Eq.
1. The three other surfaces are motionless walls, and the top of the cuboid is open and in contact with the air.

\section{Results}

Fig. 4 and 5 are a sample of the velocity profiles with the use of the ratio wall velocity to the thickness of the mixer v/W. The planes of reference are plane $X Y$ at $z=2.54 \mathrm{~cm}$ (Fig. 4) and plane $\mathrm{ZY}$ at $\mathrm{x}=11.43 \mathrm{~cm}$ (Fig. 5). In Fig. 5 and 6 the red color is the maximum velocity value (given by the variable $\mathrm{v} / \mathrm{W}$ ), and the blue color correspond to the minimum $0 \mathrm{~cm} / \mathrm{s}$. For the value $\mathrm{v} / \mathrm{W}=4.66 \mathrm{~s}^{-1}$ at any of the three amplitudes, the red color represents $13 \mathrm{~cm} / \mathrm{s}$, while for $\mathrm{v} / \mathrm{W}=23.66 \mathrm{~s}^{-1}$ the color on the wall represents $66 \mathrm{~cm} / \mathrm{s}$.

It can be seen that all the liquid velocity profiles have the same pattern regardless of the amplitude or the wall velocity. The profiles that look different are due to the change in the direction of the oscillating walls.
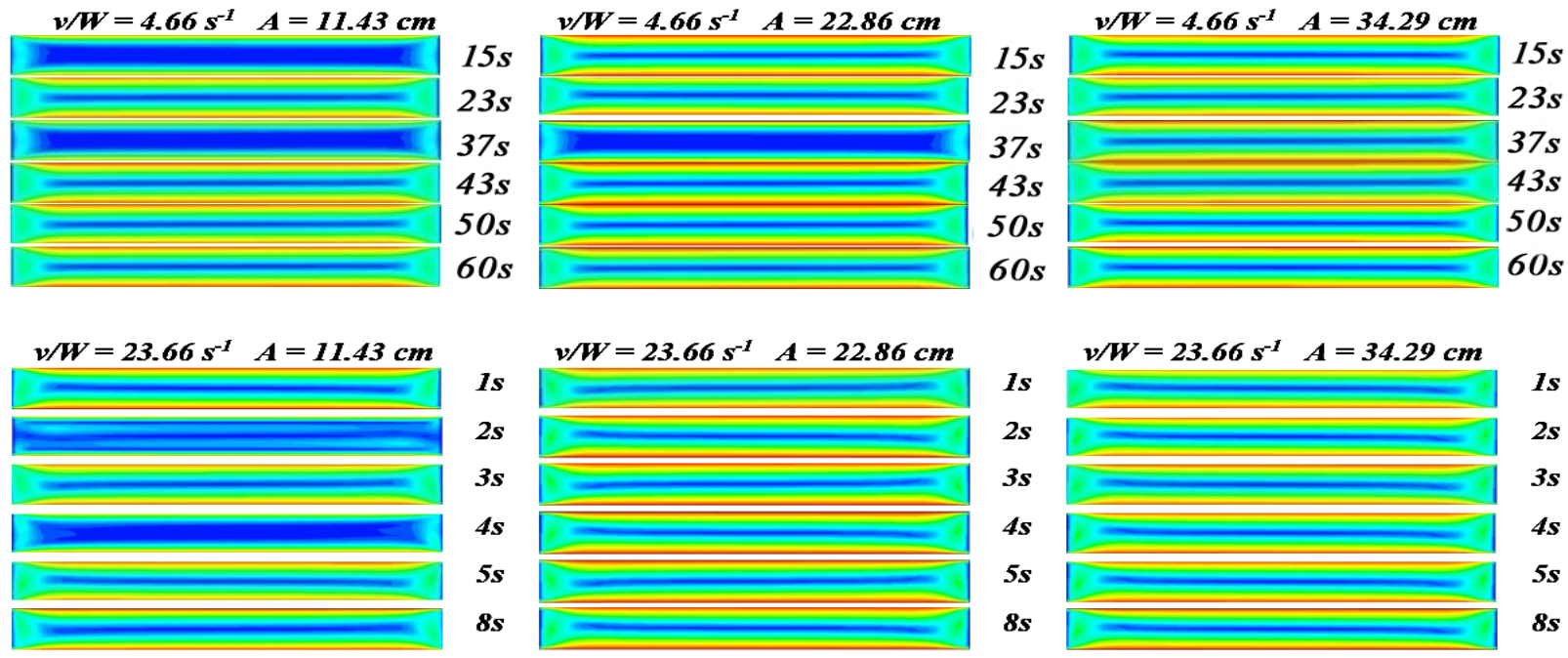

Fig. 4. Liquid velocity profiles - plane XY @ $\mathrm{z}=2.54 \mathrm{~cm}$ at different amplitudes
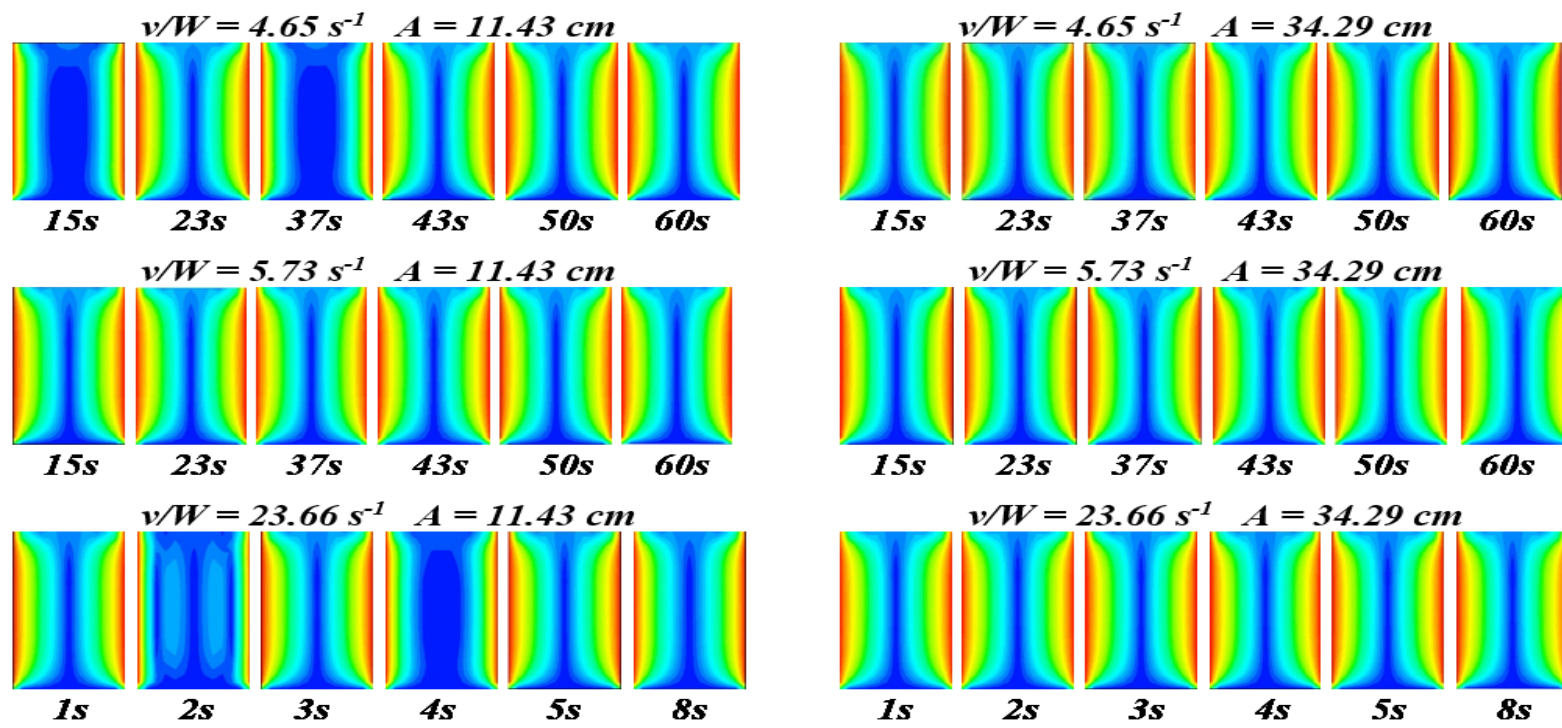

$v / W=5.73 s^{-1}$
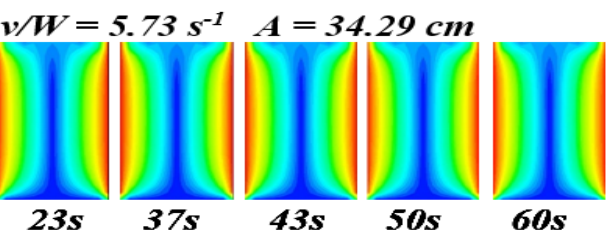

$50 \mathrm{~s}$

$v / W=23.66 \mathrm{~s}^{-1} \quad A=34.29 \mathrm{~cm}$
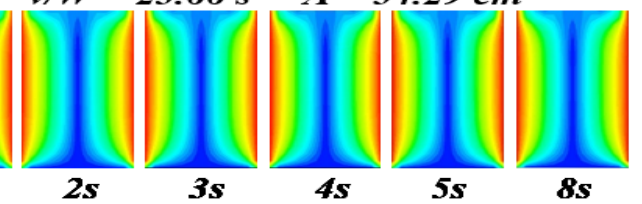

Fig. 5. Liquid velocity profiles - plane $\mathrm{YZ}$ at $\mathrm{x}=11.43 \mathrm{~cm}$ for different wall amplitudes

All the profiles present a tendency of zero velocity in the center. It happens because of the high viscosity of the liquids and the low velocity of the walls, indicating that the system is in a laminar regime. Fig. 6 and 7 shows the variation of the Reynolds number which is in the range (1.979 $0.003563)$. It is an evidence that the system is in the laminar regime. 


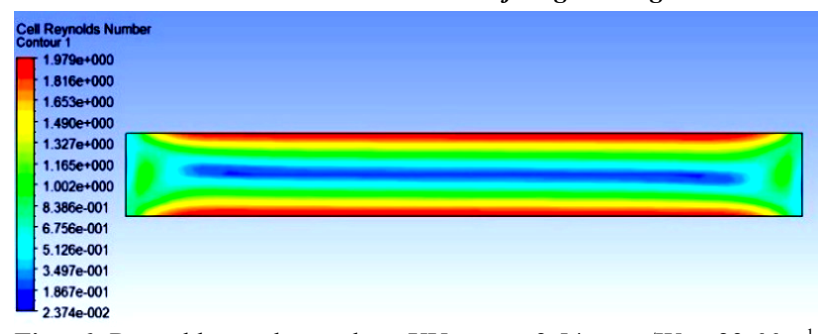

Fig. 6. Reynolds number - plane $X Y$ at $z=2.54 \mathrm{~cm}, v / W=23.66 \mathrm{~s}^{-1}$, $\mathrm{A}=34.29 \mathrm{~cm}, \mathrm{t}=4 \mathrm{~s}$

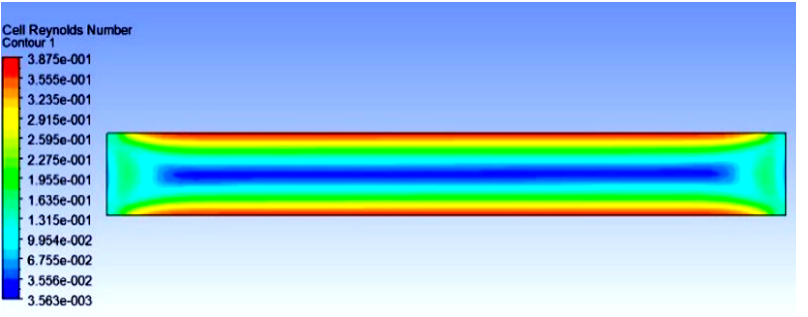

Fig. 7. Reynolds number -plane $X Y$ at $z=2.54 \mathrm{~cm}, v / W=4.66 \mathrm{~s}^{-1}$, $\mathrm{A}=11.43 \mathrm{~cm}, \mathrm{t}=30 \mathrm{~s}$

Comparing the simulations with the experimental part of mixing solids, it can be seen that the frictional regime in solids is the equivalent to the laminar regime in liquids. Although the average size of a solid is controlled at the time of mixing, these microscopically will never have the same shape and will not be organized in any specific pattern when the particles agglomerate in a container. By applying shear stress, the powders not only move in the direction where the shear is given as liquids do but also, they can move in all directions. Due to this phenomenon, the transport properties of the powder change with the time and distance causing the mixture in a shorter time interval.

Although liquid mixing is somewhat different to solid mixing, they have some similarities in their velocity profiles. Fig. 8 shows the solid mixing profile in the plane YZ. It can be seen a triangle in the center of the plane indicating the zone of no mixing which grows from the top to bottom [15, 16]. It happens because of the non-isotropy condition of the powders which allows changes in the bulk density from the wall to the center of the cavity, making possible the mixing in this area forming a triangle shape. In liquids, this situation does not happen because they have the same density. However, they have an importantparameter, the diffusion, which makes the liquid to mix completely despite the zero velocity in the center of the cavity.



Fig. 8. Solid mixing profile in the vertical layer at $t=60 \mathrm{~s}$, using the oscillating powder mixer a) $\left.\mathrm{v} / \mathrm{W}=4.66 \mathrm{~s}^{-1}, \mathrm{~A}=22.86 \mathrm{~cm}, \mathrm{~b}\right) \mathrm{v} / \mathrm{W}=4.66 \mathrm{~s}^{-1}$, $\mathrm{A}=34.29 \mathrm{~cm}$.

In the process of liquid mixing as presented in Fig. 9a and $9 \mathrm{~b}$, the mixture is given from top to bottom similar to the solid mixing. The red color symbolizes a mass fraction value of 1 for component 1 , and the blue color symbolizes a mass fraction value of 0 for component 1 . Green color indicates total mixing. The mixing profiles in the planes XY and $\mathrm{YZ}$ are similar for both liquids and solids, which means that the equations used to predict the velocity profile in liquids can be used to predict solids as well. Some parameters have to be adjusted to have a good precision.
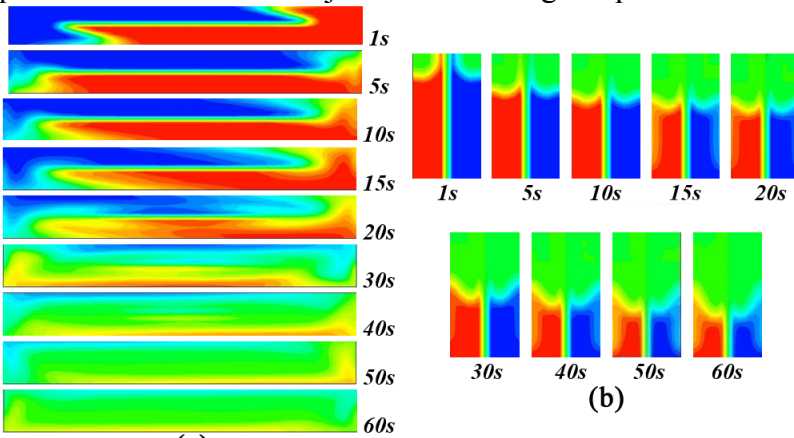

(a)

(b)

Fig. 9. Liquid mixing profile a) Plane $X Y$ at $z=2.54 \mathrm{~cm}$, $\mathrm{v} / \mathrm{W}=4.65 \mathrm{~s}^{-1}, \mathrm{~A}=11.43 \mathrm{~cm}$ b) Plane $\mathrm{YZ}$ at $\mathrm{x}=11.43 \mathrm{~cm}, \mathrm{v} / \mathrm{W}$ $=4.65 \mathrm{~s}^{-1}, \mathrm{~A}=11.43 \mathrm{~cm}$.

It can be taken the height of the zone of no mixing in plane YZ (Fig. 8 and 9b) with the specific time to predict mixing time in liquids and solids. Then, a data regression must be done, height vs. time. The corresponding data is shown in Fig. 10a and 10b. The lower the height, the higher the mixing. It can be adjusted the data to an equation of the form $H=C_{2} t^{2}+C_{1} t+C_{0}$, where $t$ is the time in seconds.

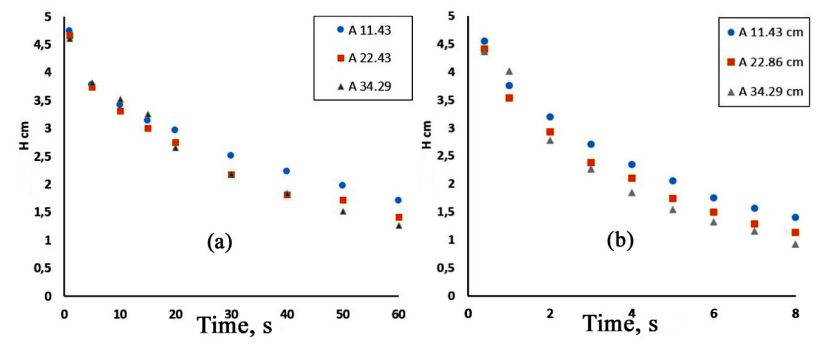

Fig. 10. Height of the no mixing zone for liquids vs. time, a) $v / W=4.66$ $\mathrm{s}^{-1}$ b) $\mathrm{v} / \mathrm{W}=23.66 \mathrm{~s}^{-1}$

Fig. $10 \mathrm{a}$ and $\mathrm{b}$ follows the same pattern. However, the total mixing time is lower for high velocities than for low velocities. A height of $2 \mathrm{~cm}$ is achieved in $5 \mathrm{~s}$ when working with $\left(\mathrm{v} / \mathrm{W}=13.66 \mathrm{~s}^{-1}\right)$ and $50 \mathrm{~s}$ for $\left(\mathrm{v} / \mathrm{W}=4.66 \mathrm{~s}^{-1}\right)$ at any wall amplitude. However, it can be seen in the same Fig. that the higher the amplitude, the lower the height.

\section{Conclusions}

The comparative study of mixing two different liquids using 3D simulation in a CFD software with an experimental part of mixing two solids in an oscillating shear mixer was done.

The comparison of the mixing process shares many similarities such as the mixing pattern which happens from the top to the bottom of the mixer.

The velocity profile obtained at the top of the mixer that causes the best and fastest mixing happens due to the nonfriction given by the air.

Under the same conditions of wall velocity, it was obtained a laminar regime in liquids, and the frictional regime in powders. In liquids, the high value of the viscosity and the low working wall velocity causes a low mixing which is improved with the help of the diffusion until reaching a homogeneous mixture. On the other hand, the non-isotropy of the powders makes them behave chaotically 
when applying small shear forces with the movable walls producing an increase in the porosity of the granular material causing a better powder distribution resulting in a high mixing. The absence of a diffusion mechanism is counteracted with the dispersion of particles due to the increment in the bulk porosity. A zone of no mixing is obtained in liquid and solids, which decreases as the time go on. Under laminar regime conditions, the Navier Stokes equations can be applied to the powders because the velocity profiles obtained are similar.

\section{Acknowledgment}

The authors appreciate the financial support of COLCIENCIAS and the Universidad del Atlántico.

This is an Open Access article distributed under the terms of the Creative Commons Attribution Licence

\section{References}

[1] C. Mayer-Laigle, C. Gatumel, and H. Berthiaux. Mixing dynamics for easy flowing powders in a lab scale Turbula $\mathbb{R}$ mixer. Chemical Engineering Research and Design, 95(0), 248261, 2015.

[2] K. Slettengren, P. Heunemann, O. Knuchel, and E. Windhab, "Mixing quality of powder-liquid mixtures studied by nearinfrared spectroscopy and colorimetry", Powder Technology, 278(0), 130-137, 2015.

[3] O. Scheibelhofer, N. Balak, D. Koller, and J. Khinast, "Spatially resolved monitoring of powder mixing processes via multiple NIR-probes", Powder Technology, 243(0), 161-170, 2013.

[4] A. Vanarase, M. Alcalá, J. Jerez, F. Muzzio, and R. Romañach, "Real-time monitoring of drug concentration in a continuous powder mixing process using NIR spectroscopy", Chemical Engineering Science, 65(21), 5728-5733, 2010.

[5] J. Vercruysse, M. Toiviainen, M. Fonteyne, N. Helkimo, J. Ketolainen, M. Juuti, T. De Beer, "Visualization and understanding of the granulation liquid mixing and distribution during continuous twin screw granulation using NIR chemical imaging", European Journal of Pharmaceutics and Biopharmaceutics, 86(3), 383-392, 2014.

[6] B. Alchikh-Sulaiman, F. Ein-Mozaffari, and A. Lohi, "Evaluation of poly-disperse solid particles mixing in a slant cone mixer using discrete element method", Chemical Engineering Research and Design, 96(0), 196-213, 2015.

[7] M. Alian, F. Ein-Mozaffari, and S. Upreti, "Analysis of the mixing of solid particles in a plowshare mixer via discrete element method (DEM)", Powder Technology, 274(0), 77-87, 2015.

[8] S. Pantaleev, S. Yordanova, A. Janda, M. Marigo, and J. Y. Ooi, "An experimentally validated DEM study of powder mixing in a paddle blade mixer," Powder Technology, vol. 311, pp. 287-302, 2017.
[9] S. Dartevelle, "Numerical modeling of geophysical granular flows: A comprehensive approach to granular rheologies and geophysical multiphase flows", Geochemistry Geophysics Geosystems, Volume 5(8), 1-28, 2004.

[10] M. Massoudi, "A Mixture Theory formulation for hydraulic or pneumatic transport of solid particles", International Journal of Engineering Science, 48(11), 1440-1461, 2010.

[11] W.-T. Wu, N. Aubry, J. F. Antaki, and M. Massoudi, "Flow of a fluid-solid mixture: Normal stress differences and slip boundary condition," International Journal of Non-Linear Mechanics, vol. 90, pp. 39-49, 2017.

[12] P. Jop, "Rheological properties of dense granular flows", Comptes Rendus Physique, 16(1), 62-72, 2015.

[13] C. Liao, S. Hsiau, J. Li, and C. Tai, "The influence of gravity on dynamic properties in sheared granular flows", Chemical Engineering Science, 65(8), 2531-2540, 2010.

[14] C. Liao, S. Hsiau, and P. Chang, "Bottom wall friction coefficients on the dynamic properties of sheared granular flows", Powder Technology, 270, Part A(0), 348-357, 2015.

[15] L. Obregon, A. Realpe, C. Rinaldi, and C.Velazquez, "Mixing of granular materials. Part I: Effect of periodic shear", Powder Technology, 197(1-2), 9-16, 2010.

[16] L. Obregon, A. Realpe, and C. Velazquez, "Mixing of granular materials, part II: effect of particle size under periodic shear", Powder Technology, 201(3), 193-200, 2010.

[17] R. Treybal, "Mass-Transfer Operations" Third ed., McGraw-Hill Book Company, 1980.

[18] R. Byron, W. Steward, and W. Lightfoot, "Transport Phenomena" Second ed., John Wiley \& Sons, 2006.

[19] H. Versteeg, and W. Malalasekera, "An Introduction to Computational Fluid Dynamics: The Finite Volume Method", Pearson Education Limited, 2007. 